\title{
ENABLERS TO BOOST BLOCKCHAIN ADOPTION IN EU
}

\author{
Artemis Voulkidis ${ }^{1}$, Theodore Zahariadis ${ }^{1,2}$, \\ Andreas Papadakis ${ }^{3}$ and Charalambos Ipektsidis ${ }^{4}$ \\ ${ }^{1}$ Synelixis SA, Greece, \\ ${ }^{2}$ National and Kapodistrian University of Athens, Greece \\ ${ }^{3}$ School of Pedagogical and Technological Education (ASPETE), Greece \\ ${ }^{4}$ Intrasoft International SA, Luxembourg
}

\begin{abstract}
This paper describes a framework to facilitate the adoption of the Blockchain technology and streamline the development of decentralised applications (DAPPs). It describes four enablers, as self-contained core modules, offering specific, key functionality using the Blockchain technology. The enabler functionality includes a) Blockchain-based ID management allowing for authentication and authorization, b) the storage of data in the IPFS distributed filesystem with guarantees of data integrity and authenticity, c) the trustworthy registration of entities, services, and bindings, $d$ ) the performance of trustworthy negotiations towards external marketplaces with the support of the Blockchain. The design and interactions of the enablers are described using sequence diagrams. The usage of the functionality provided by the enablers is also being evaluated. In parallel, we present the application of the Blockchain technology, mainly in the context of EU project Block.IS in three economic areas agriculture, finance, and logistics. We provide and discuss a digest of the decentralised applications designed and developed over a period of approximately 3 years (2019-2021). Key areas of interest, processes, workflows, and assets where Blockchain technology has been applied are described. Findings, in terms of Blockchain application, challenges and technical selections as well as third-party tools are also identified and discussed.
\end{abstract}

\section{KEYWORDS}

Blockchain, Enablers, Ethereum, Added Value Applications, Decentralized Application, Smart Contracts.

\section{INTRODUCTION}

Blockchain is considered a disruptive technology in multiple economic activities [1]. The adoption of Blockchain opens new horizons in application development ([2] and [3]) but at the same time presents a set of inherent challenges, due to its highly innovative nature and the frequent changes in the available tools. In this work, we describe our efforts and lessons learnt to support the adoption of Blockchain technology in three key areas of the European economy, namely agriculture, finance, and logistics, in the context of the EU H.2020 Block.IS project [4].

Considering the technological difficulties of adopting the Blockchain technology on behalf of SMEs (Small and Medium Enterprises), due to the inherent complexity of the technology and the volatility of the tools, we have designed a set of technological 'enablers.' These are considered self-existing, reusable modules offering specific functionality. Four enablers are described in terms of their operation, and usage, covering the areas of a) ID management, authentication, and authorization, b) immutable data storage in distributed file systems, c) trustworthy entity, service and bindings registrations, and d) trusted negotiations using external marketplaces. This 
functionality and the corresponding enablers (or similar functional modules) have been largely embraced by the participating SMEs (experimenters). Through their, almost 3-year usage (20192021), we have identified issues and challenges that have resulted in improvements and enhancements. These have included a) the need to easily deploy and connect with both private and public chains while requiring minimal underlying infrastructures, b) the ways to deal with the current heterogeneity in the Blockchain landscape, in terms of chains, tools, frameworks and applications employed.

In parallel, we provide a high-level breakdown of the innovative ideas and functionalities implemented mainly in the context of the Block.IS project leverages the Blockchain capabilities in the three, thematic areas. We also refer to the findings, the challenges and the lessons learned from the overall ecosystem, including also information on the Blockchain selections, the types of applications, and the $3^{\text {rd }}$ party tools used.

The structure of the document is as follows: In Section 2, we discuss the economic areas selected and the challenges in the adoption of the new technology. Section 3 describes the enablers, and their operations as well as the considerations and improvements performed in response to feedback. Section 4 includes the thematic digest of the Blockchain applications. The paper closes in Section 5 with the discussion and conclusions.

\section{State OF THE ART}

\subsection{Selected Economic Sectors}

A prerequisite for sustainable economic growth is the leverage of innovation and digital intelligence. Three of the most important sectors of EU economy include agrifood, logistics, and financial services. EU's agrifood sector has a strong global competitive position as the largest food and drink exporter with a market share of 18\% [5]. The interest in the link between food and health has changed mainstream consumption patterns towards valorisation of quality aspects related to good health. With long global value chains, consumers, and processors face challenges in verifying the accuracy of data from farm to table. Consumers pay premiums for products that provide this information credibly such as organic food. Current solutions focus on certifications and regulations which are costly, hard to enforce, and can be confusing to consumers. The challenge is to streamline transfer of and access to data within the sector for all actors as well as consumers ([6] and [7]).

The logistics, sector amounts to 14\% of EU's GDP, and it is expected to grow by $40 \%$ by 2040 [8]. This sector includes the distribution of goods, from raw materials up to the finished products. Contemporary supply chains span over hundreds of stages and dozens of geographical locations. This makes it hard to trace events or investigate incidents (traceability) through precise and costeffective actions limited in scope. Fraud can take place, as effectiveness of enforcement is limited. Moreover, a large degree of documentation from various sources is needed to meet regulations (e.g., import/export), which often represent a bottleneck and create costs. The challenge is to make provision and verification of information efficient along supply chains while ensuring transparency, security, and accountability ([9] and [10]].

The financial sector makes up to $20-30 \%$ of total service market revenue and about $20 \%$ of the total gross domestic product in EU economies [11]. Many financial services are data-heavy and fault-prone, thus requiring middlemen for mediation (trust), and thus entailing transaction costs. Moreover, information sharing is limited even where synergies can be realized as in the insurance industry (data sharing). The challenge is to lower operational costs for a globally integrated 
International Journal of Network Security \& Its Applications (IJNSA) Vol.14, No.1, January 2022

industry through operational simplification, regulatory efficiency improvement, settlement time reduction, liquidity, and capital improvement ([12], [13] and [14]).

\subsection{Blockchain Technology and Challenges}

Blockchain technology has been recognised as a promising and disruptive technology enabling a decentralised, trusted, and programmable way to transfer value and information [15]. It can support provenance, transparency, traceability, efficiency, trust, and data sharing, while it can be used to trade assets through digital tokens. Such tokens can represent any type of underlying asset: value, facts, quality, comments. Common uses are tokens as digital currency (monetary value), digital equity (shareholder value), tokens representing physical assets, and utility tokens that regulate access to services. As no central authority is needed to facilitate the exchanges and network nodes run the necessary checks and transactions, approved via a community consensus mechanism. Blockchain validation is thus direct since every node has access to all transactions, which are cryptographically verified and stored on the distributed ledger [16].

Adopting the Blockchain can increase the competitiveness of their users/adopters, offering products and services with enhanced value. However, the introduction of such a sophisticated technology faces technological (and other) barriers, including lack of acquaintance with this technology. To support the building of new applications, it is an existing practice to offer key functionality in a read-to-use manner, as 'enablers' [17]. Enablers are core building blocks around which added-value services and products can be built, and they can accelerate the development of Blockchain based applications.

\section{ENABLERS}

The enablers are self-contained modules with their functionality fully defined through their Application Programming Interfaces (APIs). The functionality they offer has a generic and reusable nature and can be applicable in different thematic domains (including the sectors selected in our ecosystem). Identifying such functionality and implementing it in a reusable way has been the first 'stake' in our work. Four key enablers have been designed, implemented, and provided, which are described in the next sections.

\subsection{Identity Management with AAA}

The Identity Management with AAA (Authentication, Authorization and Accounting) support allows for user identity management, authentication, authorization, and accounting based on uPort. The enabler operates between the user and the protected application or Decentralized Applications (DAPPs). The entities employed include the MetaIdentity Manager smart contract, the Proxy smart contract, and Relay SC (inspired by the uPort reference architecture [18]).

The user can interact with a) an external service (i.e., not implemented within the Blockchain as a DAPP) and b) a target DAPP where the enabler is used for authentication as well as relaying the interactions towards the target DAPP.

As presented in Figure 1, in the scenario involving the external service (non-interactive with the Blockchain), the client (the user) interacts with the external service to authenticate (interaction 1). The service invokes the enabler to perform authentication (2). The public address of the user that signed the request (using the Hashed-Based Message Authentication Code - HMAC) is known to the blockchain infrastructure. The enabler contacts the MetaIdentity Manager (3) to verify the identity of the user. 


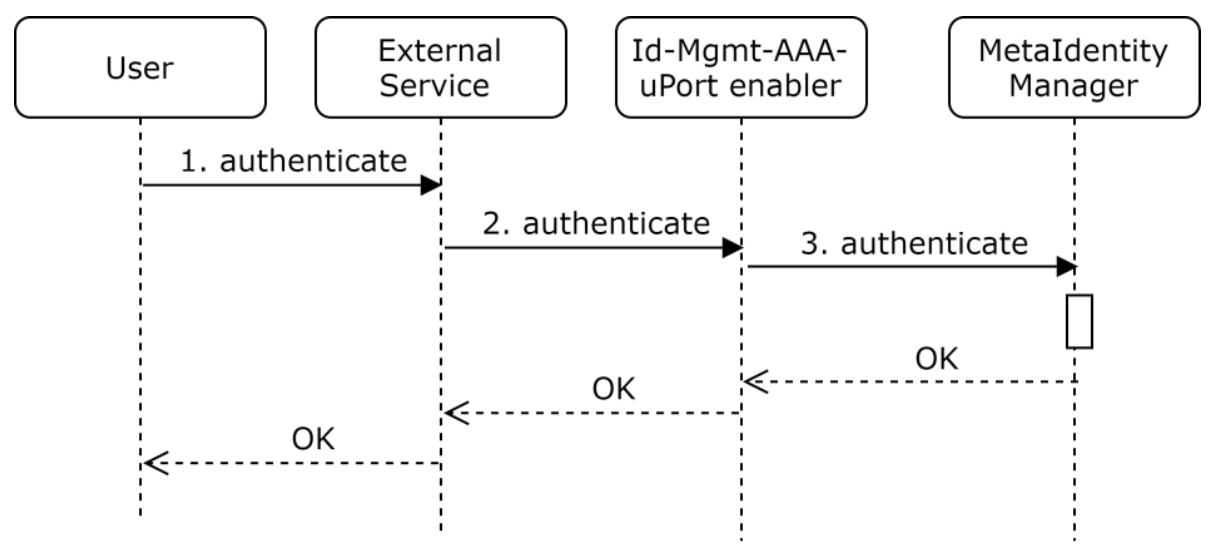

Figure 1. ID management enabler, non-interactive scenario

In the more complex scenario of interacting with a DAPP (Blockchain smart contract), the client contacts the enabler (interaction 1). The enabler invokes the Relay smart contract transferring a) the signed message containing the address of the target DAPP, the method of the target DAPP to be executed and the relevant parameters, b) the signature properties, c) the public address of the user, and d) the address of the MetaIdentity Manager Smart Contract (2). The sequence is presented in Figure 2.

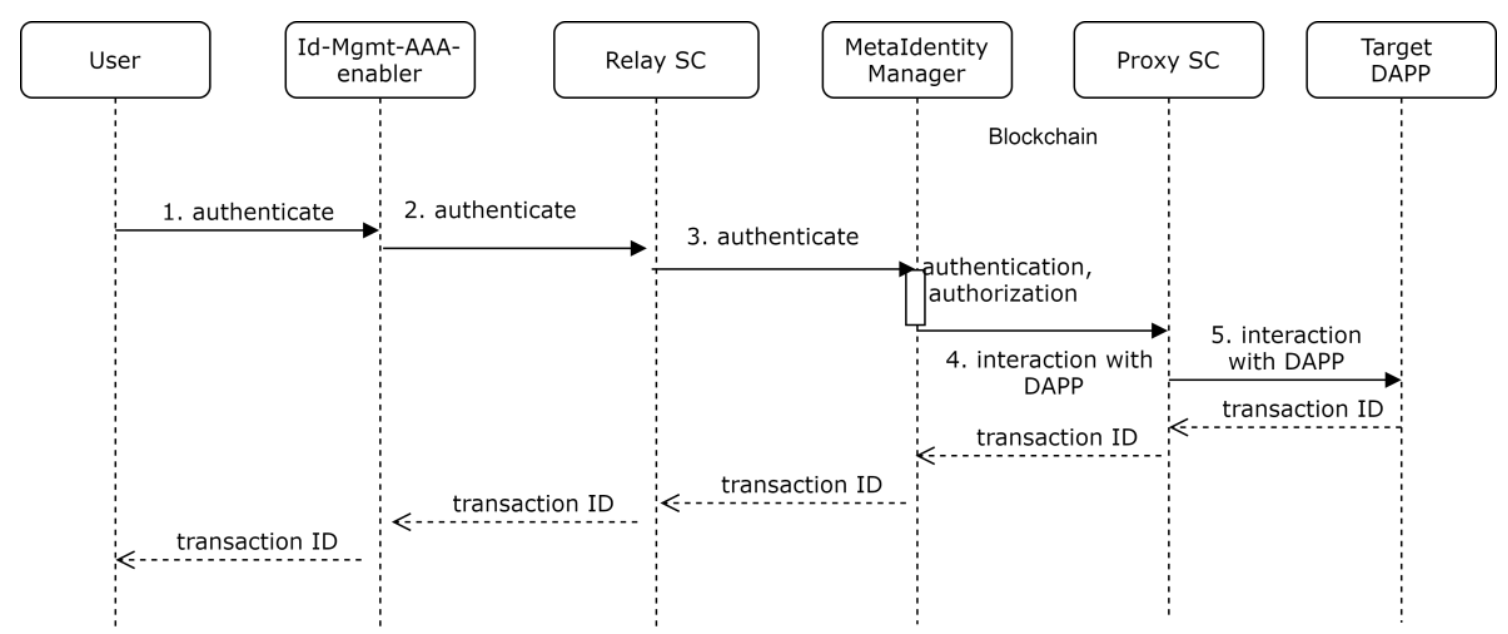

Figure 2. ID management enabler, interactive scenario

The Relay smart contract validates the integrity of the message (compatibility of the signature elements with the message hashed data) and forwards the message to the MetaIdentity Manager smart, contract (3). The MetaIdentity Manager smart contract checks the validity of the user address performing authentication and authorization. If the request is both authenticated and authorized, it is relayed to the appropriate Proxy SC (4). The Proxy smart contract forwards the message (including the method and parameters) to the target DAPP (5).

\subsection{Storage Enabler}

The Storage enabler allows storing and encrypting data (including files of increased volume, for example consisting of photos and videos) in the distributed file system Interplanetary File System (IPFS, [19]). As the files are stored in the file system, their size is not limited (by the typical 
limitations of the Blockchain infrastructure), while the information exchanges are registered in the Blockchain, becoming permanent, immutable, and traceable. The enabler is combined with a smart phone application (Storage App), which can prepare the messages including the multimedia (image and video) or other content and digitally sign the message before sending it to the enabler.

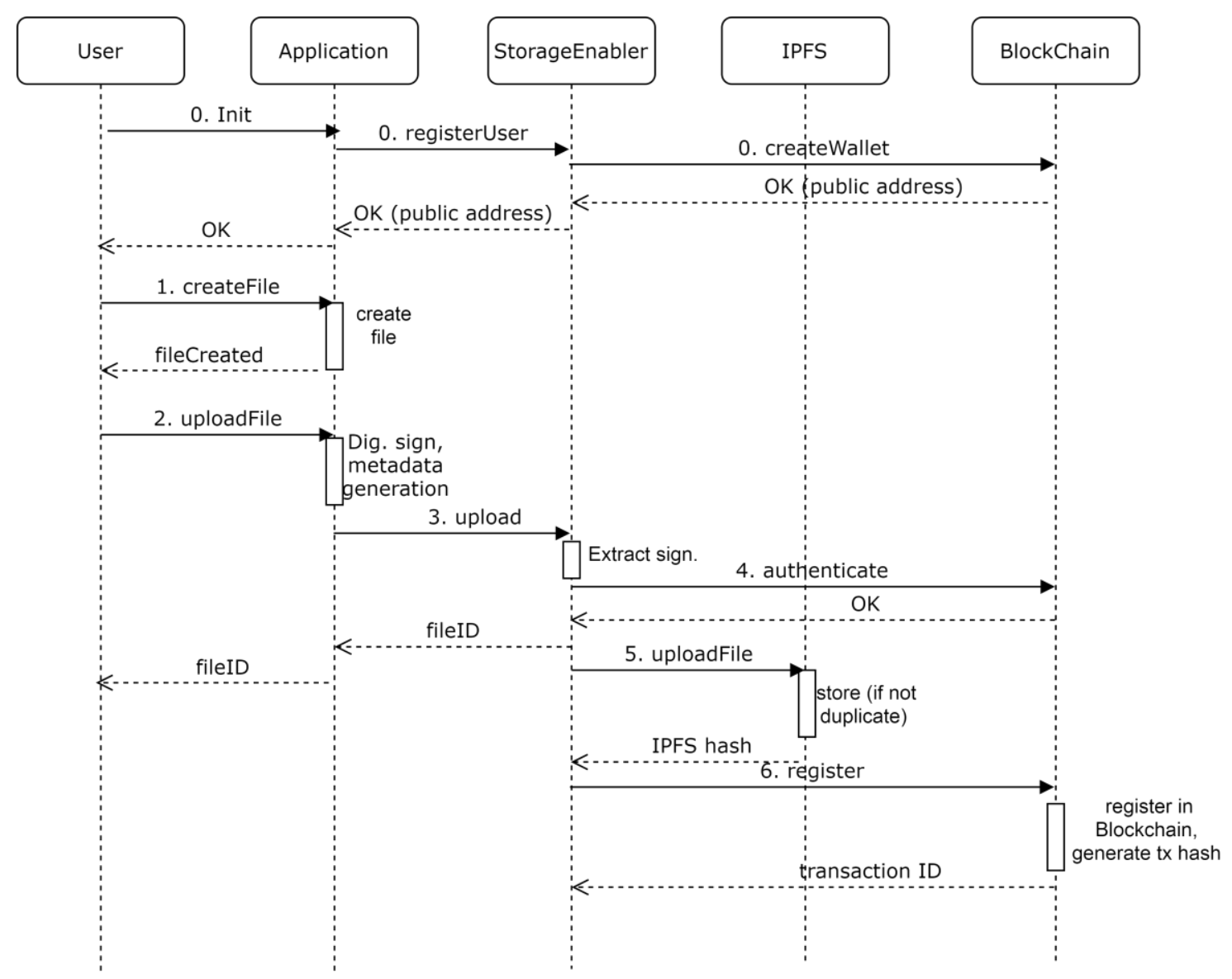

Figure 3. Storage enabler

As depicted in figure 3, the user is initially registered on the Blockchain, so that a wallet and a public address are created (interaction 0 ). The user creates the content to be uploaded using the smart phone application, e.g., by taking a photo (1). The user instructs the mobile app to prepare and upload the message, including the Blockchain address of the sender and other optional parameters such as the timestamp and the location (2). The mobile app authenticates the user, calculates the MD5 hash of the message and the SHA3 256 hex string of the MD5 hash. It unlocks the Ethereum wallet of the user, asking for his password, and signs the Secure Hash Algorithm 3 (SHA3) hash with the private key of the user. It then locks the Ethereum wallet of the user and uploads the file to the Storage enabler (3).

Using the Blockchain (4), the enabler extracts and verifies the signature parameters and performs authentication and authorization of the user. For this step, the ID management enabler can be used (as performed by the ID management enabler). The enabler then uploads the file to the IPFS file system in encrypted chunks (5). The IPFS hash is returned to the enabler, and it can be used to retrieve the file from the IPFS. The IPFS hash is registered in the Blockchain infrastructure, and a transaction ID is generated (6). The integrity and authenticity of the file can be verified at any time through the IPFS hash. 
International Journal of Network Security \& Its Applications (IJNSA) Vol.14, No.1, January 2022

\subsection{Service Registry Enabler}

The Service Registry enabler implements an entity, service, and smart contract registry directory where the participating entities can register themselves. The entity can also register and advertise the services and smart contracts it offers based on keywords (tags). This way finding services and smart contracts in Block.IS is facilitated. The concepts and approach of UDDI (Universal Description, Discovery, and Integration) as a centralised service for maintaining configuration information and naming are adapted [20].

The users of the service registry include the service provider and the service consumer. The former is offering services and DAPPs, and the latter is searching for services and DAPPs. The service (or Smart Contract) provided by the Service Provider is represented as a service structure (as described in UDDI definitions) which includes information on the type and the usage of the service. The binding templates model the actual implementation of an offered service (or Smart Contract) and include information on its access and usage. The following sections describe two types of interactions: (a) registration and deletion and (b) inquiry.

In the registration interface, as depicted in Figure 4, the Service Provider registers as an entity (1). Upon registration of the entity the enabler makes a related registration in the Blockchain (and retrieves the transaction ID). Then the entity, can register its services and bindings (for brevity they are not depicted in the figure). The registrations can be deleted (2) but not edited due to the immutable nature of Blockchain registrations.

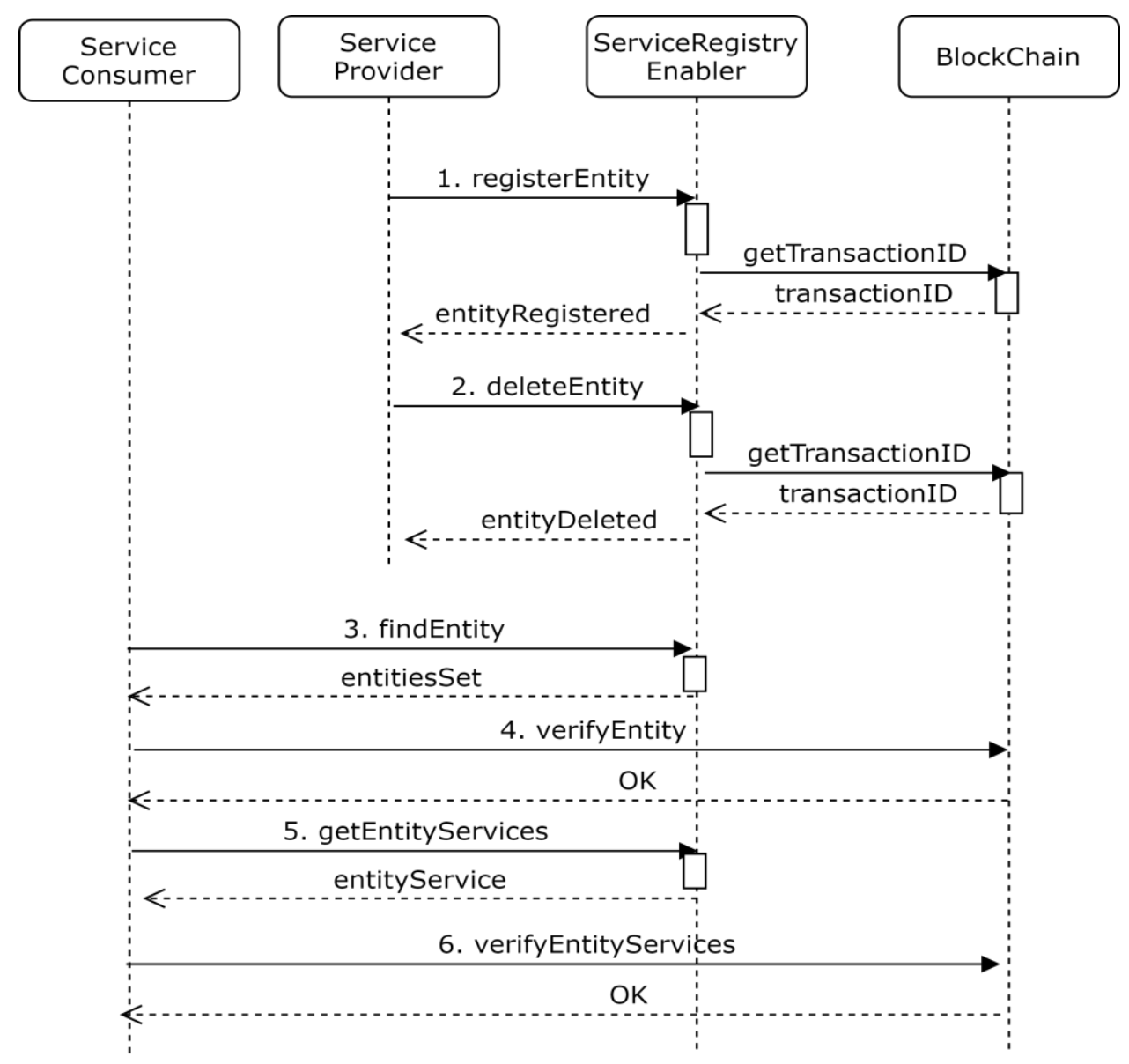

Figure 4. Service registry enabler 
International Journal of Network Security \& Its Applications (IJNSA) Vol.14, No.1, January 2022

The inquiry interface is used when an entity is searching for entities and their services to consume. Initially, the service consumer searches for entities (3). The details of the entity can be verified (using the transaction ID) with the Blockchain (4). Then the consumer can ask for the services of a specific entity (5). In a similar manner, the service details can be verified with the Blockchain (6).

\subsection{Trusted Negotiations Enabler}

The Trusted Negotiations enabler supports and enhances (in terms of integrity, authentication and non-repudiation guarantees) the critical operations taking place during the interactions with a Marketplace. Such operations are primarily related to the registration of information and include the making of an "offer", a "bid", as well as the establishment of bilateral agreements between parties. The Trusted Negotiations enabler offers integrity, authenticity, tracing, and tracking. The enabler allows the activation of external services when a DAPP event (e.g., agreement breakage or revocation) takes place.

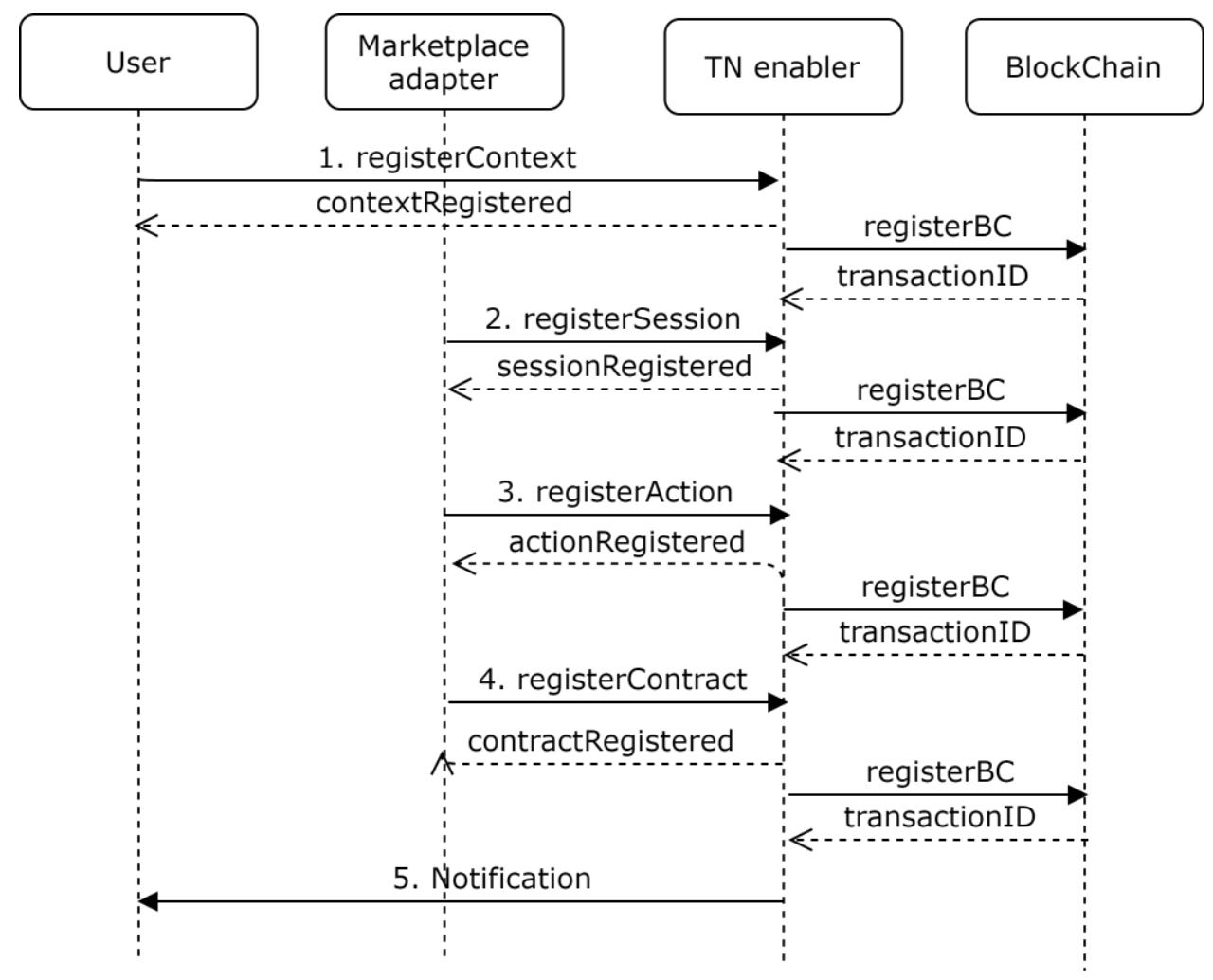

Figure 5. Trusted negotiation enabler operations.

As depicted in Figure 5, the user (acting as a Marketplace Manager) initiates the marketplace activities, providing parameters such as the type of the marketplace, auction or booking type, as well as the mechanism for accepting bids. The initiation parameters are stored in the Trusted Negotiations enabler and subsequently registered in the Blockchain infrastructure (1). A new session is registered by the marketplace adapter in the enabler and the Blockchain (2). In the context of this session, actions (offers, biddings and related metadata such as title, description, geolocation, price, creation date, duration, state) are registered in the enabler and the hash in the Blockchain (3). If the marketplace identifies that the conditions are met, the marketplace adapter registers a contract (4). In the case of peer-to-peer marketplace, the activities can be closed by the 
offeror or automatically through specific conditions. The enabler can notify the user (and other external entities) of the important events through a Pub/Sub mechanism (5).

\subsection{Enabler Usage and Improvements}

The enablers have been offered and used for a period of almost 3 years by a group of 40 external stakeholders / innovators. The provision of the enablers is performed through a set of software gitlab groups. Each group offers links to technical documentation, dynamic self-documentation services (Swagger framework), docker images and recipes. The enabler usage percentages, as depicted in Figure 6, provide an indication of the interest in the related functionality.

\section{Relative Usage of the Enablers}

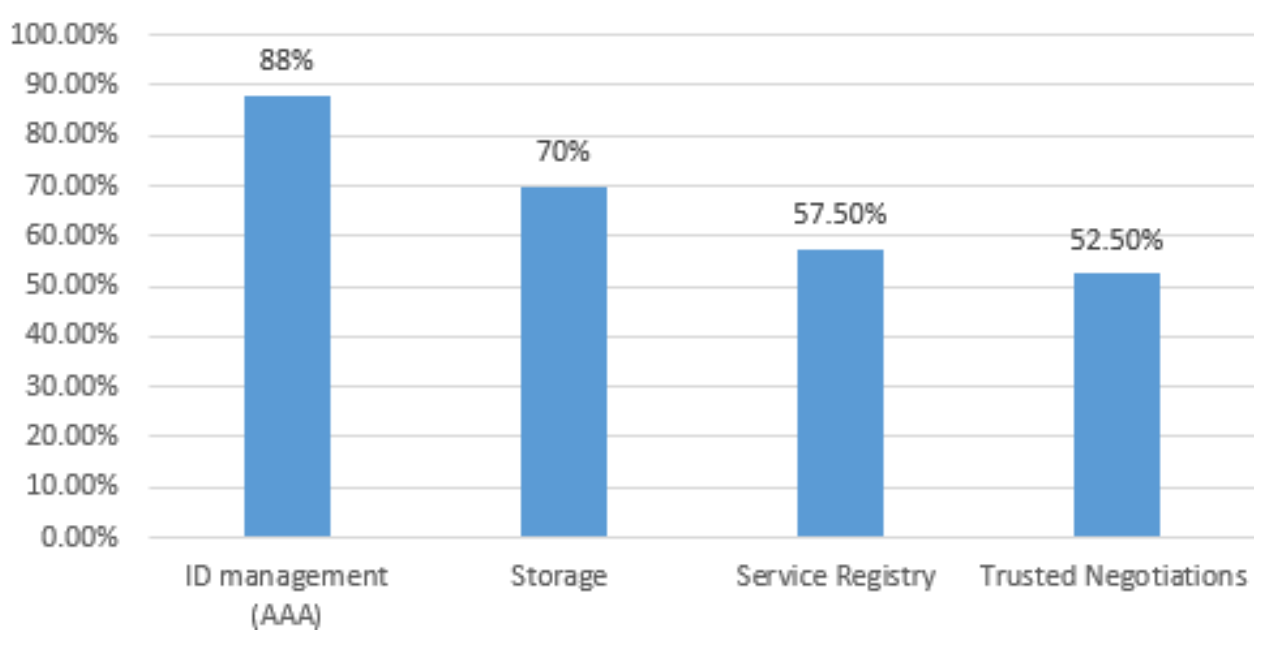

Figure 6. Enablers relative usage (percentages).

The usage of the enablers indicates the coherent selection of functionality and the reusable implementation. The most popular enabler has been the ID management enabler, which is justified due to the ubiquitous nature of the corresponding functionality. The Storage enabler comes second, the Service Registry third, and the Trusted Negotiations enabler fourth as it can be considered vertical.

In the following we discuss some key aspects, extending beyond typical maintenance tasks, such as the update and upgrade to support current tool versions and right management adaptation (minimizing the rights for executing underlying components).

User Blockchain onboarding: The usage of the enablers presupposes that the user has a public Blockchain wallet. In the absence of this, the supporting application generates a key pair (public and private) that is used for signing requests to the enabler.

From private to public Blockchains: The initial deployment of the enablers has been based on a private Blockchain (Ethereum) environment. There has been a need to connect to the public networks (for experimentation or actual transactions). This involved accommodating a full Ethereum node to connect with the public networks (mainnet and or testnets), which has been resource consuming; a first level solution has been to include a light sync node, i.e., a node that does not perform full synchronization with the network. 
International Journal of Network Security \& Its Applications (IJNSA) Vol.14, No.1, January 2022

Simplification of the connection with the public Blockchain: The connection with the public network has called for further simplification. Towards this direction, we have used intermediate platforms that perform the connection through Gateways that support the execution of Ethereum transactions. The adaptation of the enablers included a module transforming typical transactions (transferred through the local node) into raw transaction (transferred by the Infura Gateway ${ }^{1}$ ).

Heterogeneity of solutions and interoperability: The dynamic nature of chains and tools provides choices for different environments. In some cases, due to increasing gas cost or for other reasons (such as binding to specific solutions), there has been a need to change the chain used. To smooth these transitions, a standard-based approach has been followed with EVM-compatible smart contracts. Other solutions could include federated systems to be fully open and secure at the same time [21].

\section{BlockChain-Based Added Value Applications}

\subsection{Thematic areas}

In this section we present the thematic areas of the piloting projects. The steps followed include the selection of tools (including the enablers to be used), the selection of Blockchain, the specification and implementation of the Business Logic of the application, the management of data, testing and piloting, handling interoperability aspects and porting to other Blockchains, and evaluation and auditing.

Starting with the financial area, smart contracts have been designed to support processes that are currently performed with human intervention and are prone to delays, different interpretations and even disputes. These include the collateral payments for Credit Default Swap (CDS) agreements as well as non-manual cryptocurrency trading. The lifecycle of loan management, including payments, defaults, restructuring, and other credit events has also been implemented using smart contracts. Invoice management, including creation, verification, finance request, and payment, has been implemented using smart contracts. Another example has been voucher management including exchange and redemption in participating retailers, supporting crossselling and facilitating automation in the transactions.

Collaborative editing and exchange of documents, as intermediate results, or final products of design processes (such as engineering and/or architectural design) by multiple stakeholders, involve Intellectual Properties Management (IPR) issues and restrictions. The Blockchain-based solution, along with Id management, supports fine-grained procedures related to versioning, exchanging, branching, and updating (in compatibility with the editing environments).

Short- and long-term agreements have also been modelled and implemented using smart contracts. For the former, the full lifecycle of freelancing task offerings using Blockchain smart contracts includes the description of the offering (task description, budget, deadlines, relevant skills), the acceptance, the execution, the completion, and the dispute resolution. Real estate market has also been a field for the implementation of decentralized apps. Digital representation has facilitated investing and selling and secondary reselling of properties. In the clothing industry, digital representations have been created for garments and raw material.

The key aspect in finance, has been the directness on the execution, based on the smart contract, the facilitation of the management, the trustworthiness of the information, the transparency along

\footnotetext{
${ }^{1}$ Infura Development Suite, https://infura.io/
} 
with privacy and the drastic decrease of disputes. The solutions have in principle based upon private Blockchains.

In the area of logistics, physical assets have been identified (e.g., returnable transport packaging) to facilitate their management and balancing procedures using smart contracts. Such smart contracts record the availability of the physical assets and the balance updates. In addition there have been applications focusing upon the transformation / digitalization Processes for transportation systems, including the management, the exchange, and the processing of transportation documentation (such as CMR documents). These are currently handled in a manual way (often involving mistakes and omissions). Another example has been the support of decentralized vehicle sharing with a community of drivers using Blockchains. In food supply, maintaining and monitoring, in a trustworthy manner, appropriate conditions in the storage and transport of sensitive and perishable products has been a well-known problem. Inappropriate temperature levels, poor air quality, humidity, light and other conditions have a negative impact on the delicate nature of foods. Blockchain has been used to track in near real-time the digital history of product, registering transport and storage events. Registered data can come from the IoT or be included manually.

Agriculture is characterised by the need for transparency and trustworthy information, related to the ingredients used, the treatment, the allergens, the usage of herbicides and fertilizers. Current solution based on product certifications are under questioning and Blockchain appears as a valid alternative. The Blockchain applications have been focused mainly on the traceability aspects to increase the trust towards the consumer. Registered data can come from IoT platforms, supported by data entry on behalf of the processors. An important asset identified has been secondary information such as reviews, the comments, the evaluations expressed by consumers.

Food laboratory testing results have also been considered assets, and dapps have been built to support the management (including selling / reselling) of such tests in a Marketplace, using Ethereum smart contracts and the W3C Verifiable Credentials framework [22].

Other Blockchain implementations include the lifecycle management of event tickets, including issuance, selling, and reselling. Blockchain allows ticket representation as a non-fungible token (NFT), with embedded business logic / rules governing its entire lifecycle. Another example has been the management, utilization, and exploitation of multimedia content, in the context of a content marketplace platform involving creators, owners and users. Ethereum ER-20 tokens can be used for storage, payment systems, and rewards.

The area of charity has also attracted the interest for designing decentralized processes, imposing transparency through tracing the contribution (money and goods) from the donor to the receiver. The workflow includes the fund-raising, the purchase, donation of goods and receipt by the nonprofit organization and their use. Food donations in local commerce, have used local cryptocurrency to replace current practices of food banks, paper food vouchers, pre-paid debit cards, direct money transfers.

The areas and assets involved in decentralized application are depicted in Table 1. 
Table 1. Physical and non-physical (intangible) assets

\begin{tabular}{|l|l|}
\hline Physical Assets & Non-physical (intangible) \\
\hline Charity donations (money and goods) & Credit default swap \\
\hline Garment and raw material & Documents (collaborative editing with IPR) \\
\hline Event tickets & Electricity load balancing agreements \\
\hline $\begin{array}{l}\text { Food items (conditions during } \\
\text { transportation and storage) }\end{array}$ & Key management and recovery \\
\hline $\begin{array}{l}\text { Primary production in agriculture (olive } \\
\text { oil, vegetables, wine) }\end{array}$ & Loan management \\
\hline Real estate & Long term agreements and contracts \\
\hline Returnable transport packaging & Vouchers \\
\hline
\end{tabular}

\subsection{Blockchain Selections and Third-Party Tools}

The selection of blockchains has been based upon the intrinsic application requirements (public or private infrastructure), the performance requirements and the tolerance towards the volatility and increasing tendency of the transaction fees. There have also been constraints due to the regulatory frameworks (especially in finance) and the binding with technical sandboxes imposed by public agencies and/or stakeholders. The most frequent selection of Blockchain has been Ethereum (and OpenEthereum), especially for the support of smart contract, the inherent EVM compatibility and its overall acceptance. Corda R3 has primarily been used for financial applications. Hyperledger for private deployments and VeChain and EOSIO have also been used (mainly for ensuring low transaction fees). One of the main challenges identified has been the need for interoperability, pursued through smart contract (EVM) compatibility and adopting interoperable chains and parachains (such as PolkaDot Moonbeam [23]) as well as bridging solutions (such as Polygon). The need for porting applications among Blockchains for better performance and lower gas price has also been observed.

Blockchain technology has, by design, specific features that may not cover the requirements of certain application classes (such as performance constraints and support for storing heavy data). There is also the need to associate with physical assets and procedures. In this view, Blockchain can act in a complementary way with other platforms and tools. Third party tools used include decentralised filesystems, such as IPFS to support decentralized storage and management and immutability in conjunction with the Blockchain (as pursued in the Storage enabler as well). The Big chain DB, a database with blockchain characteristics, supports decentralization, immutability, and blockchain-style permissioning. It supports both public and private deployments.

Compatibility with physical components has also been considered including the POS equipment (Point of Sale) to communicate a business transaction between the client and the retailer/business owner (e.g., in the case of commercial transactions). QR codes, used by smart phone applications, can bind cyber and physical worlds. Low energy communications modules, such as Bluetooth Low Energy accompanied with single-board computer (e.g., Raspberry Pi) are used in the edge to retrieve data from scanners. In addition, IoT infrastructures, accompanied by Geographical context and map-based filtering, have also been sources of data registered in the Blockchain. 
International Journal of Network Security \& Its Applications (IJNSA) Vol.14, No.1, January 2022

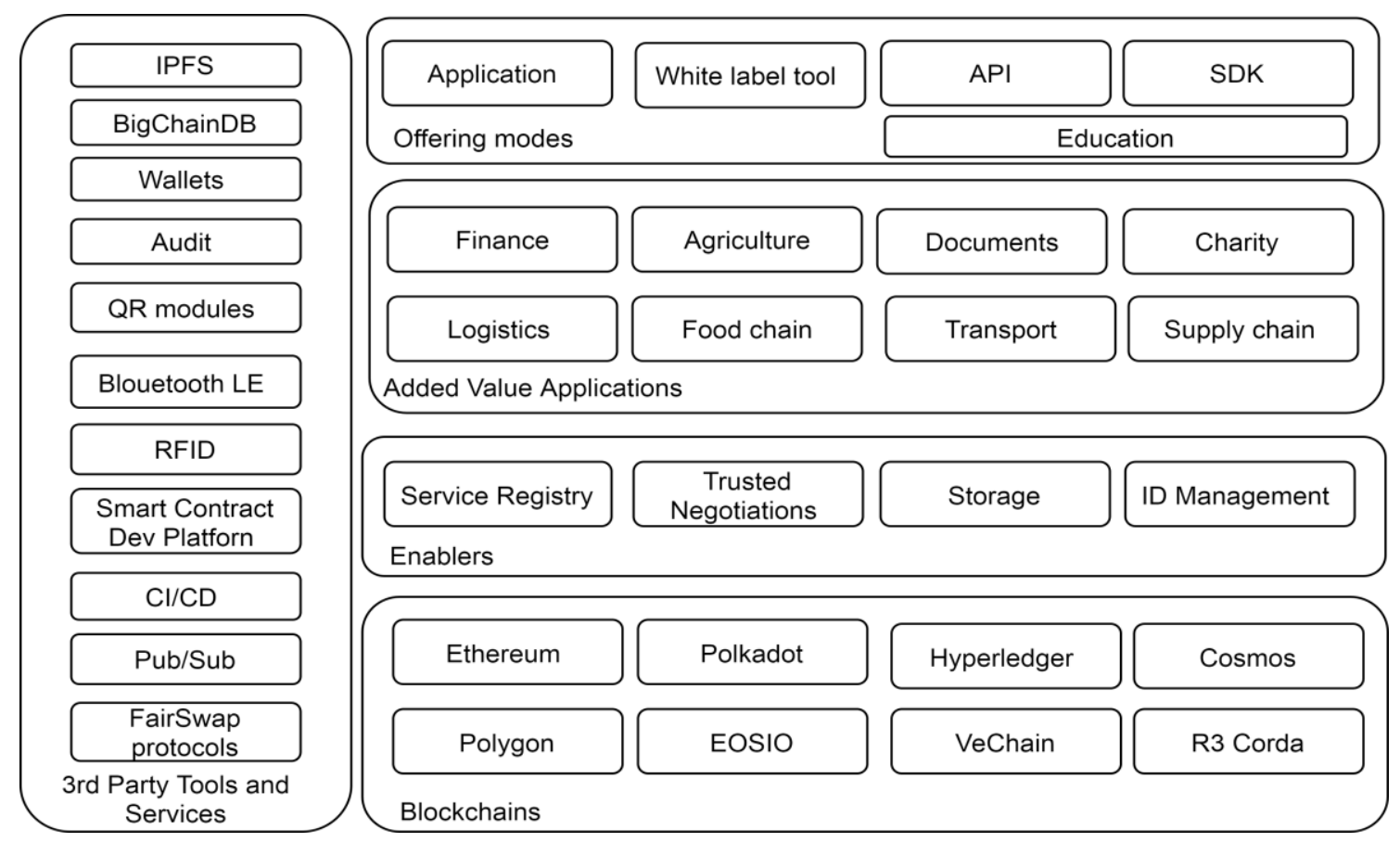

Figure 7. Value added applications development ecosystem

Figure 7 depicts the ecosystem where the value-added applications are developed, including the Blockchains selected, the enablers, the $3^{\text {rd }}$ party tools and the offering modes.

The developed functionality is provided in multiple ways. Specifically, a) as an application (web and/or mobile) for end users, b) through an API (Application Programming Interface) for developers, and c) as SDK (Software Development Kit) for further development. Specific white label offerings have been included for specific modules. Another option has been the provision of educational and simulation material (including the blockchain application and the physical components, such IoT components).

\section{CONCLUSiOnS}

The focus of this paper has been twofold. First, we have recognized the challenges in the adoption of the Blockchain technology. To alleviate the technological difficulties, we have designed and implemented four Blockchain enablers. These provide functionality that is often met in added value applications, including ID management with authentication, authorization and accounting support, the trustworthy storage of data, the registration of entities, services and bindings and the interactions / negotiations using marketplaces. The adoption and usage of the proposed functionality as offered by the enablers (or similar implementations) on behalf of the adopters, in the context of the Block.IS project ecosystem, has verified the added value they offer and their potential to facilitate the building of new innovative applications.

In parallel, through the interaction with the users and the evolution of the requirements, we have had the opportunity to identify and implement enhancements, including the access to public Blockchain networks, and the streamlining of the access to the Blockchain operations and functionality through state-of-the-art APIs. Implementation techniques (such as the development of the smart contracts) have also added value to the users of the enablers. As far as the end users of the applications are concerned, Blockchain has been streamlined through the widespread usage of wallets. 
International Journal of Network Security \& Its Applications (IJNSA) Vol.14, No.1, January 2022

In terms of the added-value applications, Blockchain has been employed in three thematic areas, namely finance, logistics and agriculture. In this work, we have presented and briefly discussed ideas and the thematic subareas and challenges involved. There has been a rich portfolio of innovative decentralized applications, offering trustworthiness, automating complex workflows, confronting disputes, and enhancing the trust of the end users. Operational aspects, such as fragmentation especially for long chains have been smoothed using Blockchain technology. Digital representations have been made both for a rich variety of physical and non-physical assets.

\section{ACKNOWLEDGEMENTS}

The current work has been partially funded, in the context of the H2020-INNOSUP-01 2018 Block.IS (number 824509) project, by the European Commission.

\section{REFERENCES}

[1] Frizzo-Barker, Julie \& Chow-White, Peter \& Adams, Philippa \& Mentanko, Jennifer \& Ha, Dung \& Sandy, Green, (2020) "Blockchain as a disruptive technology for business: A systematic review", International Journal of Information Management, Vol. 51, doi:10.1016/j.ijinfomgt.2019.10.014

[2] Asharaf, Sainudeen \& Adarsh, Sunil, (2017) "Decentralized computing using blockchain technologies and smart contracts: emerging research and opportunities", IGI Global, doi:10.4018/978-1-5225-2193-8

[3] Gul, M Junaid \& Rehman, Abdul \& Paul, Anand \& Rho, Seungmin \& Riaz, Rabia \& Kim, Jeonghong, (2020), "Blockchain Expansion to secure Assets with Fog Node on special Duty", Soft Computing, doi: 10.1007/s00500-020-04857-0

[4] Block.IS, EU H2020 project (2019-2021), "Catalyzing Blockchain Innovation”, Grant agreement No. 824509, www.blockis.eu

[5] European Commission, Agriculture and Rural Development, (2021), Monitoring Agri-trade Policy, MAP 2021-22.

[6] Xiong, Hang \& Dalhaus, Tobias \& Wang, Puqing \& Huang, Jiajin, (2020) "Blockchain Technology for Agriculture: Applications and Rationale", Frontiers in Blockchain, vol. 3, doi:10.3389/fbloc.2020.00007

[7] Feng, Huanhuan \& Wang, Xiang \& Duan, Yanqing \& Zhang, Jian \& Xiaoshuan, Zhang, (2020) "Applying blockchain technology to improve agri-food traceability: A review of development methods, benefits and challenges", Journal of Cleaner Production, doi:260. 121031. 10.1016/j.jclepro.2020.121031.

[8] European Parliament, (2015) "Report on logistics in the EU and multimodal transports in the new TEN-T corridors", European Parliament report.

[9] Betti, Quentin \& Khoury, Raphaël \& Hallé, Sylvain \& Montreuil, Benoit, (2019), "Improving Hyperconnected Logistics With Blockchains and Smart Contracts," IT Professional, vol. 21, no. 4, doi: 10.1109/MITP.2019.2912135.

[10] Apte, Shireesh \& Petrovsky, Nikolai, (2016) "Will blockchain technology revolutionize excipient supply chain management?” Journal of Excipients and Food Chemistry., 7 (3), ISSN 2150-2668

[11] Research And Markets, (2021) "Financial Services Global Market Report 2021: COVID-19 Impact and Recovery to 2030", Research and Markets.

[12] Vinayak, Muskan \& Pal Singh Panesar, Har Amrit \& Santos, Saulo dos \& Thulasiram, Ruppa \& Thulasiraman, Parimala \& Appadoo, S.S., (2018), "Analyzing Financial Smart Contracts for Blockchain," IEEE Cyber, Physical and Social Computing (CPSCom), doi: 10.1109/Cybermatics_2018.2018.00284.

[13] Varma, Jayanth Rama, (2019) 'Blockchain in Finance', Vikalpa: Journal for Decision Makers, 44(1), pp. 1-11. doi: 10.1177/0256090919839897.

[14] Schär, Fabian, (2021), "Decentralized Finance: On Blockchain- and Smart Contract-Based Financial Markets", Economic Research, doi:10.20955/r.103.153-74 
International Journal of Network Security \& Its Applications (IJNSA) Vol.14, No.1, January 2022

[15] Casino, Fran \& Dasaklis, Thomas \& Patsakis, Constantinos, (2018), "A systematic literature review of blockchain-based applications: Current status, classification and open issues". Telematics and Informatics, doi: 36. 10.1016/j.tele.2018.11.006.

[16] Xu, Min \& Chen, Xingtong \& Kou, Gang, (2019) “A systematic review of blockchain”, Springer Financial Innovation 5, 27, doi:10.1186/s40854-019-0147-z

[17] Salhofer, Peter (2018), "Evaluating the FIWARE Platform", Hawaii International Conference on System Sciences, doi:10.24251/HICSS.2018.726.

[18] Naik, Nitin \& Jenkins, Paul, (2020) "uPort Open-Source Identity Management System: An Assessment of Self-Sovereign Identity and User-Centric Data Platform Built on Blockchain," IEEE International Symposium on Systems Engineering (ISSE), doi: 10.1109/ISSE49799.2020.9272223.

[19] Benet, Juan, (2014), "IPFS - Content Addressed, Versioned, P2P File System". arXiv:1407.3561.

[20] Universal Description, Discovery and Integration v3.0.2 (UDDI), (2005), OASIS UDDI Specifications TC.

[21] Karila, Arto \& Kortesniemi, Yki \& Lagutin, Dmitrij \& Nikander, Pekka \& Paavolainen, Santeri \& Fotiou, Nikos \& Polyzos, George \& Siris, Vasilios \& Zahariadis, Theodore, (2018), "SOFIE: Secure Open Federation for Internet Everywhere", Network and Distributed System Security Symposium, doi: 10.14722/diss.2018.23001.

[22] Verifiable Credentials Data Model v1.1 (2021), https://www.w3.org/TR/vc-data-model/

[23] Burdges, Jeff \& Cevallos, Alfonso \& Czaban, Peter \& Habermeier, Rob \& Hosseini, Syed \& Lama, Fabio \& Alper, Handan \& Luo, Ximin \& Shirazi, Fatemeh \& Stewart, Alistair \& Wood, Gavin. (2020), "Overview of Polkadot and its Design Considerations", Cryptology ePrint Archive, Report $2020 / 641$.

\section{AUTHORS}

Dr. Artemis Voulkidis is the Technical Director at Synelixis Solutions SA. He holds his Dipl.-Ing. Degree in Electrical and Computer Engineering since 2007, his M.S. Degree in Technoeconomics since 2010 and his $\mathrm{PhD}$ degree since 2013 on autonomous wireless communications with emphasis on Wireless Sensor Networks, all from National Technical University of Athens. He has been actively participating in various national or European R\&D projects since 2008, while he is currently involved as senior researcher in EUfunded projects in the area of cloud computing, smart energy systems and Future Internet technologies within the FP7 and H2020 frameworks including FI-PPP and 5G-PPP. He has a strong scientific background in autonomous, game theoretic optimization and clustering techniques for Wireless Sensor Networks. The results of his scientific research have been published in high-impact journals of the ACM and IEEE communities, while he also provides reviews for ACM and IEEE journals and conferences.

Dr. Theodore Zahariadis (male) is the Chief Technical Officer of Synelixis. He was granted a Ph.D. degree in electrical and computer engineering from the National Technical University of Athens, and a Dipl.-Ing. Degree in computer engineering from the University of Patras. Currently, Th. Zahariadis is the Technical Coordinator of H2020 DEFEDER and PHOENIX projects. He has been the Project Coordinator of the H2020 COSSIM project and the FP6 project ASTRALS, the Project Technical Coordinator of many H2020 and FP7 projects. In the past, he has been with Ellemedia Technologies as the CTO, the Hellenic Aerospace Industry (HAI) as Chief Engineer, the Lucent Technologies/ Bell-Laboratories, Holmdel, NJ as a Senior Consultant, and Intrasoft / Intracom. Since 1994, he has participated in many ACTS, ARTEMIS and IST/ICT projects as Senior Researcher or Technical Manager. Within 2019, Dr. Zahariadis was also announced Full Professor at the National and Kapodistrian University of Athens (NKUA). He has published more than 150 papers in magazines, journals and conferences and he is the author/editor of 5 books. He has been a reviewer and principal guest editor in many IEEE and ACM journals and magazines and has served as TPC of a number of IEEE Conferences and has more than 2500 citations. Since 2001, he is an evaluator of IST/ICT proposals and reviewer/rapporteur in many IST projects. He is a member of the IEEE, the ACM and the Technical Chamber of Greece.

Dr. Andreas Papadakis (male) was granted a Dipl.-Ing. Degree and Ph.D. electrical and computer engineering from the National Technical University of Athens. He is a Professor in the Department of Electrical and Electronics Engineering Educators of the School of Pedagogical and Technological Education (ASPETE). His research interests include Internet and telecommunications systems and services design and provision. He has been working for the private sector, mainly in RTD, with more than 20 years 
International Journal of Network Security \& Its Applications (IJNSA) Vol.14, No.1, January 2022

of experience, assuming the role of project and/or technical coordinator and project manager. He has also worked in the public sector in the Greek Ministry of Interior, contributing to the design of the national interoperability framework and the PKI infrastructure. He has more than 50 publications in International Conferences and Journals and edited 3 books. He regularly contributes as reviewer in International Conferences and Journals. He is member of Technical Chamber of Greece.

Mr. Charalampos (Babis) Ipektsidis (Male) is a delivery manager, and dissemination and research and innovation policy expert. He has more than 14 years of experience in R\&D\&I and his expertise and research interests are in research and innovation policy, impact assessment, science and technology policy studies, and policy analysis in general. During his career Mr. Ipektsidis has been involved as a project manager, dissemination and communication expert as well as policy expert in more than 30 projects and strategic studies including among others DIATOMIC, MaaS4EU, Block.IS, EU-COMMUNITY, INNO Policy TrendChart, "EU online Trustmarks - Building Digital Confidence in Europe", "Impact on Innovation of FP5 and FP6", "Scale and Scope as Drivers for the European Research Area". Currently, he is a member of the coordination team in the project STAR "Safe and Trusted Human Centric Artificial Intelligence in Future Manufacturing Lines". Mr Ipektsidis holds a Doctorandus/Masters Degree in International Business Studies (with specialization in Innovation Management and Marketing) from Maastricht University. He additionally holds a M.A in European Studies on Society, Science and Technology from Maastricht University, University of Namur and the European Inter-University Association on Society, Science and Technology as also a Minor in European Studies. 